\title{
Surface curvature-quantized energy and forcefield in spacetime-warped chemical physics
}

Z. R. Tian, Department of Chemistry/Biochemistry, and Institute for Nanoscience/Engineering, University of Arkansas, Fayetteville, AR 72701, USA, (Email) rtian@uark.edu.

Surface curvature-quantized energy and forcefield were introduced to support wellestablished theories, geometrize Heisenberg's Uncertainty, and quantize the gravity, gravitycounterbalancing levity and Einstein's spacetime to develop new chemical geometrophysics for quantifying geometrochemistry and geometrochemical biology on small curving surfaces.

Most recently, simple-shape particles

surface area-to-volume ratio was quantized into a geometro-wavenumber $\left(v_{\mathrm{Geo}}\right)$, or the geometro-energy $\left(\mathrm{E}_{\mathrm{Geo}}\right) \equiv \mathrm{hc} \cdot v_{\mathrm{Geo}}$, to predict and compare quantitatively the nanoparticles (NPs) and atoms size-dependent properties ${ }^{1,2}$ that thermodynamics, quantum mechanics (QM) and chemical bonding theory ${ }^{3}$ cannot help. These properties, either long-known or new, range widely from atoms' ionization potentials and electronegativity (EN) to NPs' atomistic nature, bonding energy, chemical potential of formation, redox potential, surface adsorbates' stability, and surface defects' reactivity. For countless complex- or irregular-shape NPs, molecules, and clusters whose $v_{\text {Geo }}$ values aren't easy to calculate, however, the $\mathrm{E}_{\mathrm{Geo}}$ may not work as easily. Here, let's quantize shaper corners' and edges' greater surface curvature $(1 / \mathrm{r}) \equiv$ Spacetime Wavenumber $\left(v_{\text {ST }}\right)$, i.e. Spacetime Energy $\left(\mathrm{E}_{\mathrm{ST}}\right)=h c\left(v_{\mathrm{ST}}\right)=h c(1 / r)$ (see the Fig. 3 below), where the $\mathrm{r}=$ particle radius, $h=$ Planck constant, $c=$ speed of light, and $h c \approx$ $1.24(\mathrm{keV} \cdot \mathrm{nm})$. Indeed, the smaller atoms' higher EN and smaller 0-dimensional (0D) NPs' lower melting point $\left(T_{m}\right)$ and higher (i.e. more blue-shifted) optical bandgap (ЕвG) (see the Supplementary Table S1) ${ }^{3-9}$ are all 

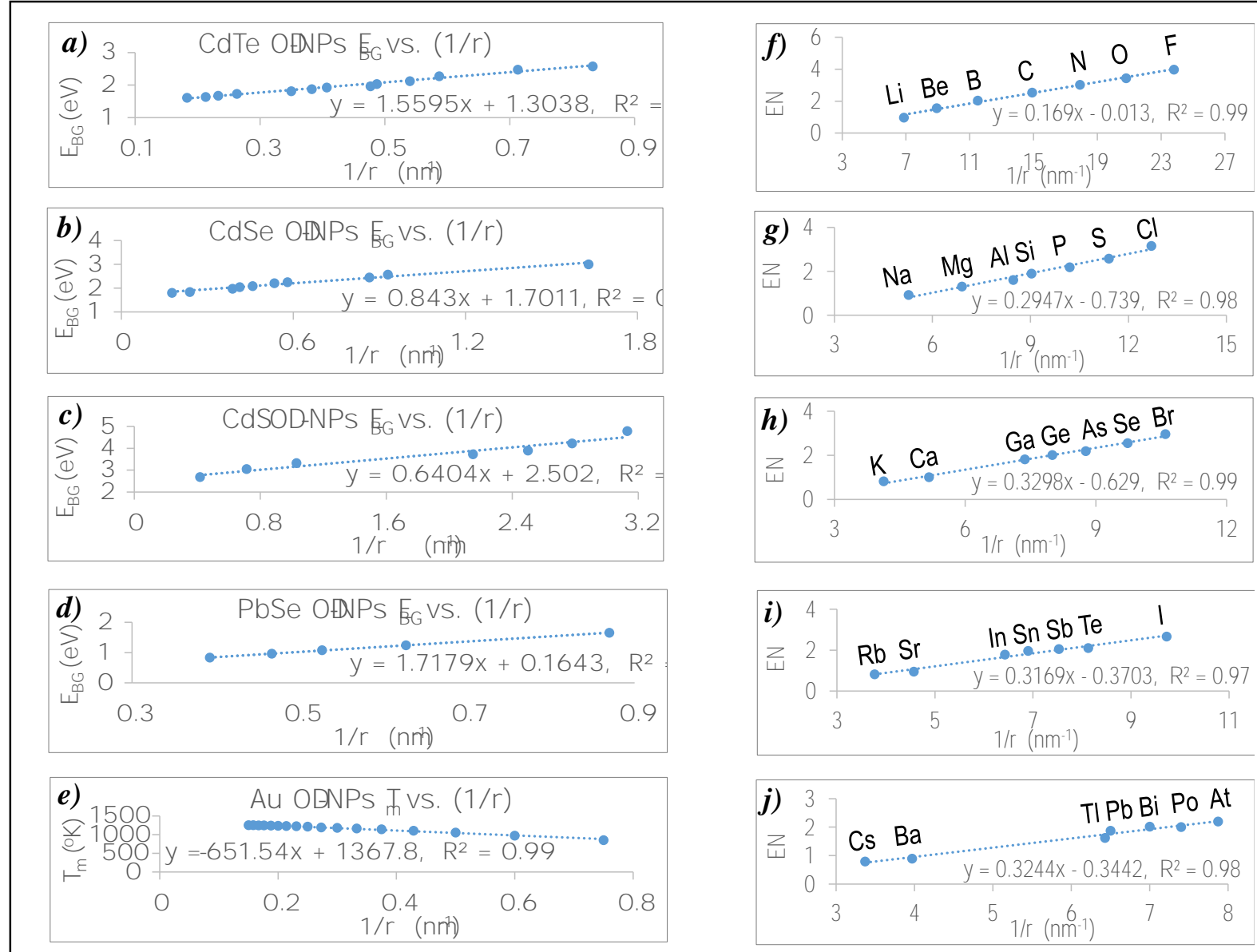

Fig. 1. The generalized linear fits of spherical particles' $3(1 / \mathbf{r})$ i.e. $3\left(\mathrm{E}_{\mathrm{ST}} / \mathrm{hc}\right)$. With $0 \mathrm{D}$ -

NPs $E_{\mathrm{BG}}(\mathbf{a})-(\mathbf{d})$, with Au 0D-NPs $\mathrm{T}_{\mathrm{m}}(\mathbf{e})$, and with main-group atoms' EN values (f)-(j).

governed linearly by their greater (1/r) (i.e.

$\left.\mathrm{E}_{\mathrm{ST}} / h c\right)$ (Fig. 1). Since a spherical particle's $\nu_{\mathrm{Geo}}=4 \pi \cdot \mathrm{r}^{2} /\left(4 \pi \cdot \mathrm{r}^{3} / 3\right)=3(1 / \mathrm{r})$, i.e. $\mathrm{E}_{\mathrm{Geo}} \equiv$ $h c \cdot v_{\mathrm{Geo}}=\mathrm{hc} \cdot 3(1 / \mathrm{r})$, or $\mathrm{E}_{\mathrm{ST}}=\mathrm{E}_{\mathrm{Geo}} / 3$, the nonspherical structure's $\mathrm{E}_{\mathrm{Geo}}$ ovresimply lumps all surface-curvatures' EsT. The EsT, proving the Surface Curvature-Energy Equivalence (SCEE), is more generalized than the $\mathrm{E}_{\mathrm{Geo}}$ to derive the $\mathrm{E}_{\mathrm{ST}}$-based chemical physics below.
For a nucleon $\left(\mathrm{r} \approx 0.4 \times 10^{-6} \mathrm{~nm}\right)^{10}$, for instance, its $\mathrm{E}_{\mathrm{ST}}=1.24 /\left(0.4 \times 10^{-6}\right)=3.1$ $(\mathrm{GeV})$, contrasting the $\mathrm{E}_{\text {(Nuclear Binding) }} \leq 10$ $(\mathrm{MeV})^{11} \quad$ (see the Supplementary Information). Thus, in a spherical particle's total energy, the $\mathrm{E}_{\text {(surface) }}\left(=\mathrm{E}_{\mathrm{ST}}=h c / r\right)$ unites the $\mathrm{E}_{\text {(potential) }}\left(=\mathrm{m}_{\mathrm{o}} \mathrm{c}^{2}\right.$, i.e. Einstein's massenergy $\left.{ }^{12}\right)$ with the $\mathrm{E}_{(\text {(knetic) }}(=\mathrm{pc}$, i.e. de Broglie's matter-wave ${ }^{13}$ energy i.e. QM), 
where the $\mathrm{m}_{\mathrm{o}}=$ particle rest mass, and $\mathrm{p}=$ particle momentum.

By the energy-force relation, the Spacetime Force $\left(\mathrm{F}_{\mathrm{ST}}\right)=\mathrm{E}_{\mathrm{ST}} / \mathrm{d}$ (see the Fig. 3), where the $d=$ distance to the curving surface. The convex surfaces' positive curvature-based repulsing $\mathrm{F}_{\mathrm{ST}(\text { convex })}>0$ (resembling a positive charge's outward forcefield), and concave surfaces' negative curvature-based attractive $\mathrm{FST}_{\text {SToncave) }}<0$ (resembling a negative charge's inward forcefield). The repulsing FST(convex) should couple with the attracting $\mathrm{F}_{\mathrm{ST} \text { (concave), like that }}$ between opposite point-charges (see the Supplementary Fig. S1), driving a smaller freestanding S-shaped string or spiral (Fig. 2a) (e.g. DNA- and $\alpha$-helices) to rotate faster.

Given a nanoshell's inner concave negative $(-1 / \mathrm{r})$-based attracting force $\mathrm{m}_{\mathrm{T}}^{\mathrm{T}} \mathrm{( \textrm {A } )}=$ $-\mathrm{hc} /(\mathrm{rd})$, thickness $y$, and outer convex positive $\{1 /(\mathrm{r}+y)\}$-based repulsing force ${ }_{\mathrm{M}} \mathrm{T}(\mathrm{R})=\mathrm{hc} /\{\mathrm{d}(\mathrm{r}+\mathrm{y})\}$, the ${ }_{\mathrm{S}} \mathrm{T}($ nanoshell $)=\left(\mathrm{M}_{\mathrm{T}} \mathrm{T}(\mathrm{R})\right.$ $\left.+{ }_{\mathrm{S}}^{\mathrm{T}(\mathrm{A})}\right)=-h c y /\left(\mathrm{r}^{2} \mathrm{~d}+\mathrm{ryd}\right)$. Symmetrically, the ${ }_{\mathrm{ST}}^{\mathrm{T} \text { (nanoshell) }}$ should couple with the atom's

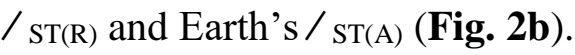

For a smaller void between closepacked smaller particles, the greater

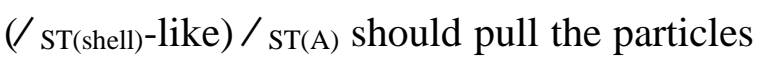
closer. This trend helps expand the electroncentered Chemical Bonding Theory ${ }^{3}$ to the $\mathrm{m}_{\mathrm{S}} \mathrm{T}$ (A)-based bonding between self-assembled NPs often with a same (or similar) surface isoelectric point, and attribute a solid planet's mass-induced gravity naturally to the voids

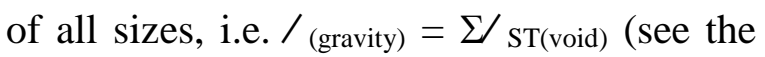

\section{Supplementary Information).}

Logically, a particle should migrate in a constant speed (or stay still) when the surrounding net ${ }_{\mathrm{T}(\mathrm{Net})}=0$, acceleratively when the ${ }_{\mathrm{T}(\mathrm{Net})}>0$, deceleratively when the ${ }_{\mathrm{S}}^{\mathrm{T}(\mathrm{Net})}<0$, and rotate when passing by another particle (Fig. 2c). This supports the $\mathrm{F}_{\mathrm{ST}}$-geometrized mechanics and dynamics in Newton's Laws of Motion and Wheeler's Geometrodynamic Gravitation ${ }^{14,15}$. 

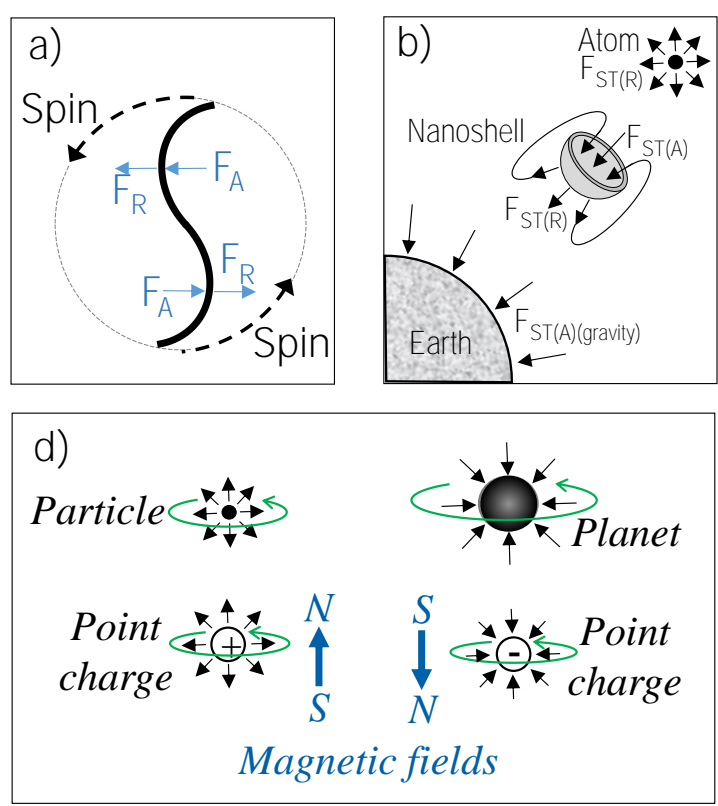

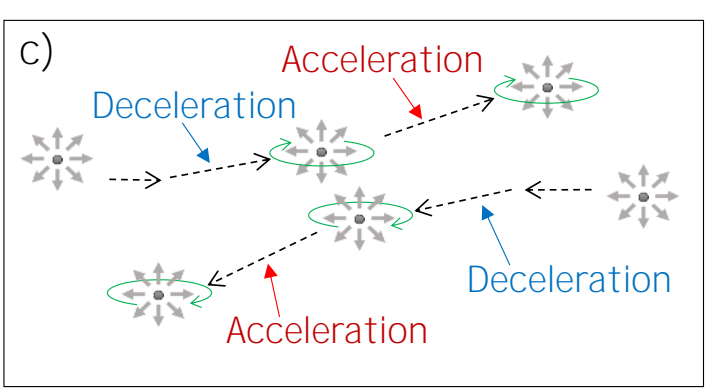

e)
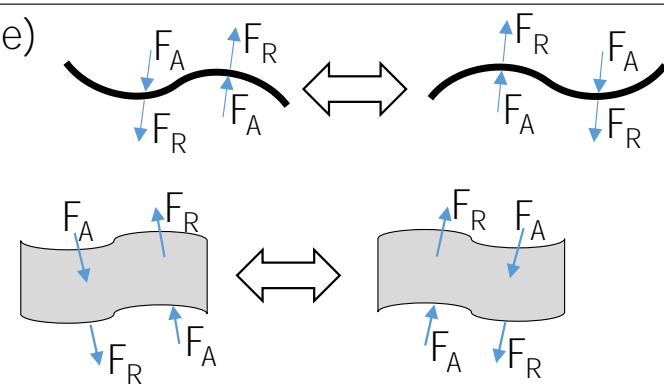

Fig. 2. Schematics of $F_{S T}$ interaction, migration, rotation, vibration and oscillation.

In between the S-shaped string's convex and concave surfaces (a), Earth, nanoshell, and atom (b), migrating particles (c), spinning particle, planet, and point-charges (d), and thermally vibrating and oscillating string's and membrane's curving surfaces (e).

Countering bigger planets' greater $\mathrm{F}_{\mathrm{A}}$ that pulls the planets closer (see the Supplementary Fig. S2), the greater $\mathrm{F}_{\mathrm{ST}(\mathrm{R})}$ should repel the smaller particles farther apart each other (Fig. 2c). This supports smaller particles higher incapability of collision (matching the Ideal Gas Law, and the particle collider's need of high energy input), faster Brownian motion (i.e. nonstop acceleration, deceleration, and rotation), lower boiling and freezing points, and greater chaosity (or Boltzmann entropy i.e. thermodynamics).

Further, a spinning particle's rotating FST should couple with an oppositely spinning particle's and with the spinning Earth's, as what spinning point-charges do (Fig. 2d). This can help expand Maxwell

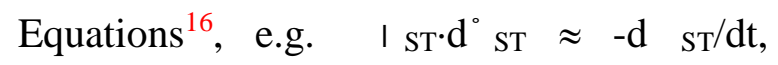

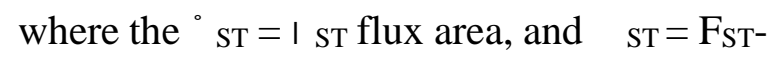
spun magnetics flux (which should be 
stronger on a spinning neutron $\operatorname{star}^{17}$ than on the lighter Earth).

Smaller particles in faster rotation, acceleration and deceleration should interact more using their greater $\mathrm{F}_{\mathrm{ST}}$, forming waves in e.g. stronger diffractions after flying through two thinner and closer slits ${ }^{18}$ (i.e. four matter-vacuum interfaces). This can help geometrize the QM's Wave-Particle Dualism $^{19}$ and quantum entanglement ${ }^{20}$.

Furthermore, thinner and longer wires and membranes can use their edge's and curving surface's higher E $\mathrm{ST}_{\mathrm{S} \text { (Levity) }}$ to energize nearby smaller particles to migrate over a longer mean free path on the edge and surface in a higher speed (or momentum). This supports the F ST $_{\text {in }}$ quicker motor proteins' migration on thinner nanowires ${ }^{21}$ and in rapid charge transfer on thousands of topological insulators $^{22}$. Further, the DNA- and $\alpha$-helices edges' EST should energize nearby ions and molecules to spiral rapidly along the edge to activate countless chemical changes, thus- expanding the chemical bonding theory ${ }^{3}$ to considering the $\mathrm{E}_{\mathrm{ST}}$ and $\mathrm{E}_{\mathrm{ST}}$-Spun $\quad$ ST. On thinner strings and membranes in higher thermal vibration and/or oscillation, their greater $\mathrm{E}_{\mathrm{ST}(\text { Surface) }}$ (Fig. 2e) can support the dynamic Tribo-energy Nanogenerators ${ }^{23}$, String Theory ${ }^{24}$, and M-Theory ${ }^{25}$.

Countering the gravity-induced light redshift ${ }^{26,27}$ (Fig. 3a), the NPs' optical bandgap blueshift (Fig. 1) characterizes the gravity-counterbalancing property i.e. levity, namely (Fig. 3b). All smaller particles' greater levity can help quantize and generalize Einstein's spacetime in all abovementioned cases, and more below.

First, the levity-contracted timeframe and levity-expanded spaceframe (Fig. 3b) support the small concave, convex and flat surfaces' spacetimes; Spacetime Conjugation i.e. $\Delta($ Spaceframe $) / \Delta($ Timeframe $) \approx-1$ when (surface-curvature) $\rightarrow \pm \infty$; and Spacetime Uncertainty or $\Delta($ Spaceframe $) \cdot \Delta($ Timeframe $)$ $\leq 0$ (Fig. 3c). This can help geometrize 

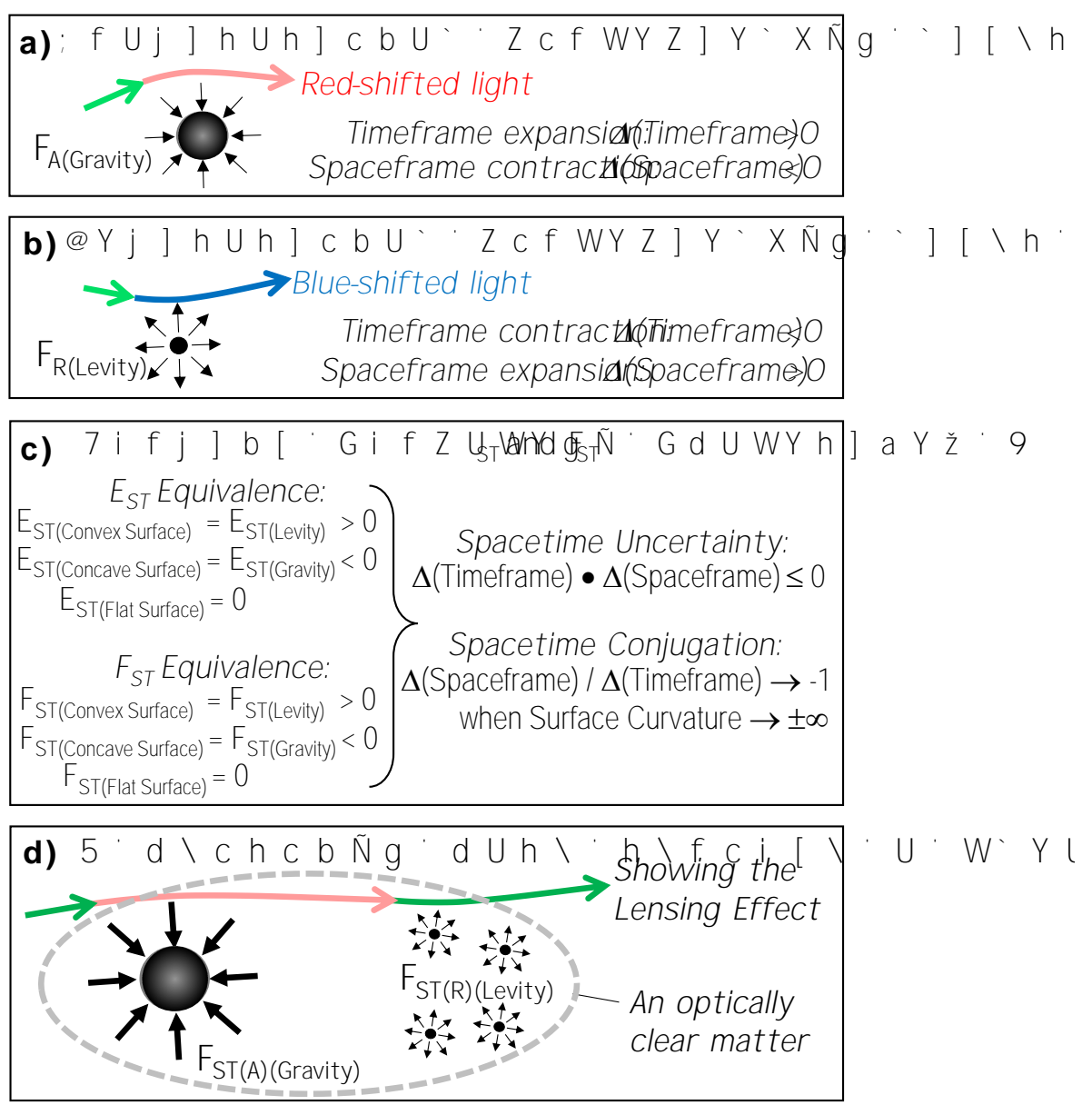

Fig. 3. The Warping Spacetimes' Energy and Forcefield for Gravity (a), Levity (b), their Uncertainty, Conjugation, and Equivalences (c), and for a Clear Matter (d).

particles' warping spacetimes in

Heisenberg's Uncertainty ${ }^{28}$. Since the matter-

and vacuum-surfaces' spacetimes warp oppositely at their interface, the $\mathrm{E}_{\mathrm{ST}(\text { levity) }}=$ $\mathrm{E}_{\mathrm{ST}(\text { convex matter surface) }}=\mathrm{E}_{\mathrm{ST}(\text { concave vacuum surface }) \text {, }}$ and the $\mathrm{E}_{\mathrm{ST} \text { (gravity) }}=\mathrm{E}_{\mathrm{ST}(\text { concave matter surface) }}=$ $\mathrm{E}_{\mathrm{ST}(\mathrm{convex} \text { vacuum)} \text {. }}$

Second, a photon shouldn't change color (or wavelength) after traveling through a group of matters along a path with the gravity-redshift(s) offsetting the levityblueshift(s), as if through a lens-like clear matter looking "invisible" (i.e. optically dark) against the dark cosmic background (Fig. 3d). Accordingly, the clear (or dark) matter's spacetime-offsetting degree tells its clearness (or darkness), and its geometry (or lens shape and size) determines its Lensing 
Effect $^{29}$. These can help advance the cosmochemistry.

Third, the Earth's gravity can couple constructively in a pinhole with the concave surface's gravity-equivalent $\mathrm{F}_{\mathrm{ST}(\mathrm{A})}$ but destructively on a pin-tip with the convex surface's levity-equivalent $\mathrm{F}_{\mathrm{ST}(\mathrm{R}) \text {. This can }}$ help quantitatively predict and compare smaller pores', tips', and edges' higher geometrochemical reactivities.

Fourth, a curving surface's FST(surface) $=\mathrm{E}_{\mathrm{ST}(\mathrm{R})} / \mathrm{r}=\mathrm{hc} / \mathrm{r}^{2}$, much like the gravitational force $\left(\mathrm{F}_{\mathrm{G}}=\mathrm{G} \cdot \mathrm{m}_{1} \cdot \mathrm{m}_{2} / \mathrm{r}^{2}\right)$ and electromagnetic force $\left(\mathrm{F}_{\mathrm{EM}}=\mathrm{kq}_{1} \mathrm{q}_{2} / \mathrm{r}^{2}\right)$, supporting the forcefield--spun magnetics. Thus, Einstein's $\mathrm{E}_{\text {(Gravity)-offsetting }}{ }^{30}$ Dark Energy $\mathrm{E}_{(\text {Dark })} \equiv$ $\mathrm{E}_{\mathrm{ST}(\text { Levity })}=\mathrm{hc} / \mathrm{r} \neq$ constant, which exists everywhere in the geometrochemistry and geometrochemical biology.

In summary, the SCEE-induced $E_{S T}$ and levity support consistently the spacetimewarped geometrochemical physics and wellestablished sciences. The warping spacetimes migration-, rotation-, vibration- and interaction-based mechanics, dynamics, kinetics, energetics, and magnetics will inspire new experimental verifications and technological innovations.

\section{EFERENCES:}

1 Tian, Z. Nanoparticles' and Atoms' Geometry-Wave Potential Unified Properties. ChemRxiv. Preprint (Sept. 5, 2019). https://doi.org/10.26434/chemrxiv.9759551.v1.

2 Tian, Z. Geometry-Wave Potential Quantified and Unified Properties in General Chemistry. ChemRxiv. Preprint (Sept. 5, 2019). https://doi.org/10.26434/chemrxiv.9759581.v1.

3 Pauling, L. The Nature of Chemical Bond (Cornell U. Press, 1960). ISBN-10: 0801403332.

4 Rossetti, R.; Nakahara, S. and Brus, L. E. Quantum size effects in the redox potentials, resonance Raman spectra, and electronic spectra of CdS crystallites in aqueous solution, $J$. Chem. Phys. 79, 1086-1088 (1983). doi.org/10.1063/1.445834. 
5 Murray, C. B.; Norris, D. J. and Bawendi, M. G. Synthesis and characterization of nearly monodisperse CdE (E = Sulfur, Selenium, Tellurium) semiconductor nanocrystallites, J. Am. Chem. Soc. 115, 8706-8715 (1993). doi.org/10.1021/ja00072a025.

6 Vossmeyer, T.; Katsikas, L.; Giersig, M.; Popovic, I. G.; Diesner, K.; Chemseddine, A.; Eychmueller, A. and Weller, H. CdS Nanoclusters: Synthesis, Characterization, Size Dependent Oscillator Strength, Temperature Shift of the Excitonic Transition Energy, and Reversible Absorbance Shift. J. Phys. Chem. 98, 7665-7673 (1994). doi.org/10.1021/j100082a044.

7 Yu, W. W.; Qu, L.; Guo, W. and Peng, X. Experimental determination of the extinction coefficient of CdTe, CdSe, and CdS nanocrystals. Chem. Mater. 15 (14), 2854-2860 (2003). doi.org/10.1021/cm034081k.

8 Moreels, I.; Lambert, K.; De Muynck, D.; Vanhaecke, F.; Poelman, D.; Martins, J. C.; Allan, G. and Hens, Z. Composition and size-dependent extinction coefficient of colloidal PbSe quantum dots, Chem. Mater. 19, 6101-6106 (2007). doi.org/10.1021/cm071410q.

9 Gold Nanoparticles: Properties and Applications, Sigma-Aldrich, (www.sigmaaldrich.com/technical-documents/articles/materials-science/nanomaterials/goldnanoparticles.html).

10 Basdevant, J.-L.; Rich, J. and Spiro, M. Fundamentals in nuclear physics (Springer, 2005). ISBN-10: 1441918493.

11 Fewell, M. P. The atomic nuclide with the highest mean binding energy, Am. J. Phys. 63 (7), 653-658 (1995). doi.org/10.1119/1.17828.

12 Einstein, A. Does the inertia of a body depend upon its energy-content? Annalen der Physik, 18, 639-643 (1905). http://www.astro.puc.cl/ rparra/tools/PAPERS/e_mc2.pdf. 
13 de Broglie, L. The Reinterpretation of Wave Mechanics. Foundations of Physics 1, 1 (1970). https://link.springer.com/article/10.1007/BF00708650.

14 Wheeler, J. A. Geometrodynamics (Academic Press, New York, 1963). LCCN 62013645.

15 Misner, C. W.; Thorn, K. S. and Wheeler, J. A. Gravitation (W. H. Freeman, 1973). ISBN 07167-0334-3.

16 Fitzpatrick, R. Maxwell's Equations and the Principles of Electromagnetism (Infinity Science Press, Hingham, MA 2008). https://www.fisica.net/ebooks/eletricidade/FITZPATRICK\%20$\% 20$ Maxwell's\%20equations\%20and\%20the\%20principle\%20of\%20electromagnetism.pdf.

17 Reisenegger, A. Origin and evolution of neutron star magnetic fields, https://arxiv.org/abs/astro-ph/0307133.

18 Schrödinger, E. Probability relations between separated systems. Math. Proceed. Cambridge Phil. Soc. 32, 446-452 (1936). DOI: https://doi.org/10.1017/S0305004100019137.

19 Einstein, A.; Podolsky, B. and Rosen, N. Can quantum-mechanical description of physical reality be considered complete? Phys. Rev. 47, 777-780 (1935). doi:10.1103/PhysRev.47.777.

20 Greiner, W. Quantum Mechanics: An Intro (Springer, 2001). ISBN 978-3-540-67458-0.

21 Berg, J. M.; Tymoczko, J. L. and Stryer, L. Biochemistry (W. H. Freeman, New York, 2002). ISBN-10: 0-7167-3051-0.

22 Gibney, E. Thousands of exotic 'topological' materials discovered through sweeping search. Nature, 560, 151-152 (2018). doi: 10.1038/d41586-018-05913-4.

23 Wu, C.; Wang, A. C.; Ding, W.; Guo, H. and Wang, Z. L. Triboelectric Nanogenerator: A Foundation of the Energy for the New Era, Adv. Energy Mat, 9, 1 (2018). doi.org/10.1002/aenm.201802906. 
24 Green, M.; Schwarz, J. and Witten, E. Superstring theory. Vol. 1: Introduction. (Cambridge University Press, 2012). ISBN 978-1107029118.

25 Witten, E. String theory dynamics in various dimensions. Nuclear Phys. B. 443, 85-126 (1995). doi:10.1016/0550-3213(95)00158-O.

26 Forbes, E. G. A history of the solar red shift problem. Annals of Science, 17(3), 129-164 (1961). doi.org/10.1080/00033796100202601.

27 Einstein, A. Die Grundlage der allgemeinen Relativitätstheorie Annalen der Physik, 49 (1916). Reprinted in English translation in The Principle of Relativity. (Dover Publications Inc, New York, 1952). http://www.synergiecomunicazione.it/eventime/Abstract.pdf.

28 Heisenberg, W. Über den anschaulichen Inhalt der quantentheoretischen Kinematik und Mechanik, Zeitschrift für Physik. 43, 172-198 (1927). doi.org/10.30965/9783846757123_004.

29 Refregier, A. Weak gravitational lensing by large-scale structure. Annual Rev. Astronomy Astrophysics. 41 (1), 645-668 (2003). https://arxiv.org/abs/astro-ph/0307212.

30 Einstein, A. Kosmologische Betrachtungen zur allgemeinen Relativitätstheorie, (Cosmological Considerations in the General Theory of Relativity) Koniglich Preußische Akademie der Wissenschaften, Sitzungsberichte (Berlin): 142-152 (1917). .http://echo.mpiwgberlin.mpg.de/ECHOdocuView?url=/permanent/echo/einstein/sitzungsberichte/S250UZ0K/i ndex.meta\&pn=1. 


\section{EXTENDED DATA}

I. Supplementary Information.

II. Supplementary Table S1.

III. Supplementary Figures S1\&S2.

IV. References.

\section{Surface curvature-quantized energy and forcefield in spacetime-warped chemical physics}

Z. R. Tian, Department of Chemistry/Biochemistry, and Institute for Nanoscience/Engineering, University of Arkansas, Fayetteville, AR 72701, USA, (Email) rtian@uark.edu.

\section{Supplementary Information:}

If six nucleons were hypothetically close-packed, the octahedral void's center-spherical radius $\mathrm{r}_{\text {(void) }} \approx 0.414 \cdot \mathrm{r}_{\text {(nucleon) }}{ }^{1}$, and $\mathrm{E}_{\mathrm{ST} \text { (void) }} \approx-(3.1 / 0.414)=-7.49(\mathrm{GeV})$, although the six nucleons' Nuclear Binding Energy ${ }^{2,3}\left(\mathrm{E}_{\mathrm{NB}}\right) \leq 0.060(\mathrm{GeV})$, i.e. $\left.\mathrm{E}_{\mathrm{NB}} \ll<\mid \mathrm{E}_{\mathrm{ST}(\mathrm{void})}\right)$. The similar from hypothetically close-packing four nucleons into a tetrahedron. Equivalently, the EsT-energized nucleons cannot be close-packed, not to mention the close-packing of the much smaller quarks. This trend supports logically the Spacetime Conjugation, Spacetime Uncertainty and Heisenberg's Uncertainty for nucleons in a nucleus, and for "massless" elementary particles in a nucleon. 
II. Supplementary Table S1a ${ }^{4,5}$ :

\begin{tabular}{|c|c|c|c|c|c|c|}
\hline \multicolumn{7}{|c|}{ Spherical (0D) NPs' (1/r), optical bandgap $\left(\mathrm{E}_{\mathrm{BG}}\right)$ data } \\
\hline \multicolumn{2}{|c|}{ (a)CdTeNPs } & \multicolumn{2}{|c|}{ (b).CdS AVPS } & (c)CdSNPS & \multicolumn{2}{|c|}{ (d).PbSAVPS } \\
\hline $\begin{array}{c}E_{B G} \\
(e V)\end{array}$ & $\begin{array}{c}1 / r \\
\left(n m^{-1}\right)\end{array}$ & $\begin{array}{c}E_{B G} \\
(e V)\end{array}$ & $\begin{array}{c}1 / r \\
\left(n m^{-1}\right)\end{array}$ & $\begin{array}{cc}E_{B G} & 1 / r \\
(e V) & \left(n m^{-1}\right)\end{array}$ & $\begin{array}{l}E_{B G} \\
(e V)\end{array}$ & $\begin{array}{c}1 / r \\
\left(n m^{-1}\right)\end{array}$ \\
\hline 2.58 & 0.83 & 3.00 & 1.63 & $4.78 \quad 3.1$ & 0.833 & 0.39 \\
\hline 2.48 & 0.70 & 2.57 & 0.93 & $4.22 \quad 2.8$ & 0.967 & 0.47 \\
\hline 2.28 & 0.60 & 2.45 & 0.87 & $3.89 \quad 2.5$ & 1.077 & 0.53 \\
\hline 2.13 & 0.53 & 2.25 & 0.58 & $3.72 \quad 2.2$ & 1.243 & 0.63 \\
\hline 2.04 & 0.50 & 2.21 & 0.53 & $332 \quad 10$ & 1.656 & 0.87 \\
\hline 1.97 & 0.47 & & 0.46 & $\begin{array}{l}1.0 \\
0.70\end{array}$ & & \\
\hline 1.93 & 0.40 & 2.05 & 0.41 & 3.05 & & \\
\hline 1.88 & 0.40 & & 0.39 & 2.68 & & \\
\hline 1.82 & 0.37 & 1.80 & 0.24 & & & \\
\hline 1.74 & 0.27 & & & & & \\
\hline 1.68 & 0.23 & & & & & \\
\hline 1.64 & 0.21 & & & & & \\
\hline 1.62 & 0.18 & & & & & \\
\hline
\end{tabular}


II. Supplementary Table S1b ${ }^{4,5}$ :

Spherical (0D) NPs' (1/r) and melting point $\left(T_{m}\right)$ data

\begin{tabular}{|cc|}
\hline \multicolumn{2}{|c|}{ (e). Au NPs } \\
\hline $\begin{array}{c}1 / r \\
\left(\mathrm{~nm}^{-1}\right)\end{array}$ & $\begin{array}{c}\mathrm{T}_{\mathrm{m}} \\
(\mathrm{oK})\end{array}$ \\
\hline 0.750 & 853.16 \\
0.600 & 973.16 \\
0.500 & 1053.16 \\
0.429 & 1103.16 \\
0.375 & 1143.16 \\
0.333 & 1163.16 \\
0.300 & 1183.16 \\
0.273 & 1193.16 \\
0.250 & 1213.16 \\
0.231 & 1223.16 \\
0.214 & 1228.16 \\
0.200 & 1233.16 \\
0.188 & 1241.16 \\
0.176 & 1245.16 \\
0.167 & 1248.16 \\
0.158 & 1251.16 \\
0.150 & 1254.16 \\
\hline
\end{tabular}




\section{Supplementary Table S1 ${ }^{4,5}$ :}

Main-groups atoms' (1/r) and EN data

\begin{tabular}{|c|c|c|c|c|c|}
\hline Atom & $1 / r\left(n^{-1}\right)$ & E N & Atom & $1 / r\left(n m^{-1}\right)$ & EN \\
\hline $\mathrm{Li}$ & 6.87 & 0.98 & $\mathrm{Rb}$ & 3.77 & 0.82 \\
\hline $\mathrm{Be}$ & 8.93 & 1.57 & $\mathrm{Sr}$ & 4.57 & 0.95 \\
\hline B & 11.5 & 2.04 & $\ln$ & 6.43 & 1.78 \\
\hline C & 14.9 & 2.55 & Sn & 6.90 & 1.96 \\
\hline $\mathrm{N}$ & 17.9 & 3.04 & $\mathrm{Sb}$ & 7.53 & 2.05 \\
\hline 0 & 20.8 & 3.44 & $\mathrm{Te}$ & 8.13 & 2.10 \\
\hline $\mathrm{F}$ & 23.8 & 3.98 & 1 & 9.73 & 2.66 \\
\hline $\mathrm{Na}$ & 5.27 & 0.93 & $\mathrm{CS}$ & 3.37 & 0.79 \\
\hline $\mathrm{Mg}$ & 6.90 & 1.31 & $\mathrm{Ba}$ & 3.97 & 0.89 \\
\hline Al & 8.47 & 1.61 & $\mathrm{TI}$ & 6.43 & 1.62 \\
\hline $\mathrm{Si}$ & 9.03 & 1.90 & $\mathrm{~Pb}$ & 6.50 & 1.87 \\
\hline$P$ & 10.2 & 2.19 & $\mathrm{Bi}$ & 7.00 & 2.02 \\
\hline$S$ & 11.4 & 2.58 & Po & 7.40 & 2.00 \\
\hline $\mathrm{Cl}$ & 12.7 & 3.16 & At & 7.87 & 2.20 \\
\hline $\mathrm{K}$ & 4.13 & 0.82 & & & \\
\hline $\mathrm{Ca}$ & 5.17 & 1.00 & & & \\
\hline Ga & 7.37 & 1.81 & & & \\
\hline $\mathrm{Ge}$ & 8.00 & 2.01 & & & \\
\hline As & 8.77 & 2.18 & & & \\
\hline $\mathrm{Se}$ & 9.73 & 2.55 & & & \\
\hline $\mathrm{Br}$ & 10.6 & 2.96 & & & \\
\hline
\end{tabular}


III. Supplementary Figures:

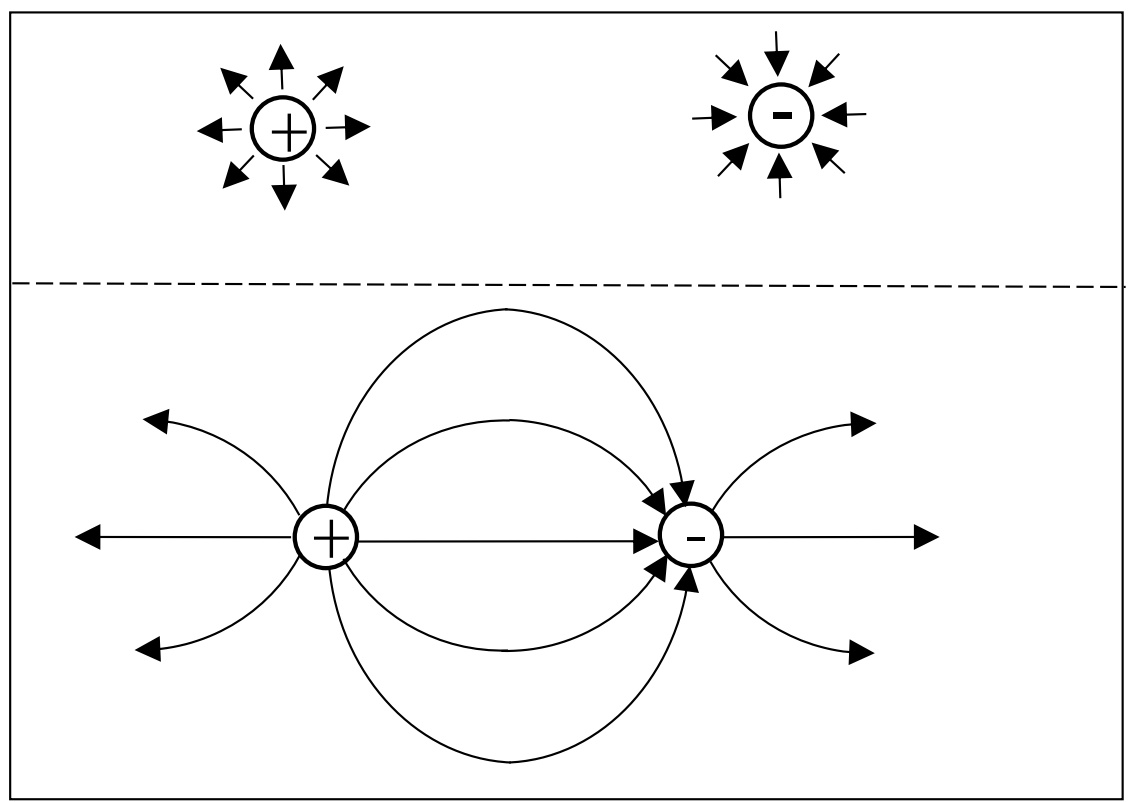

Fig. S1. Positive point-charge's outward forcefield, negative point-charge's inward forcefield, and the two forcefields' coupling.

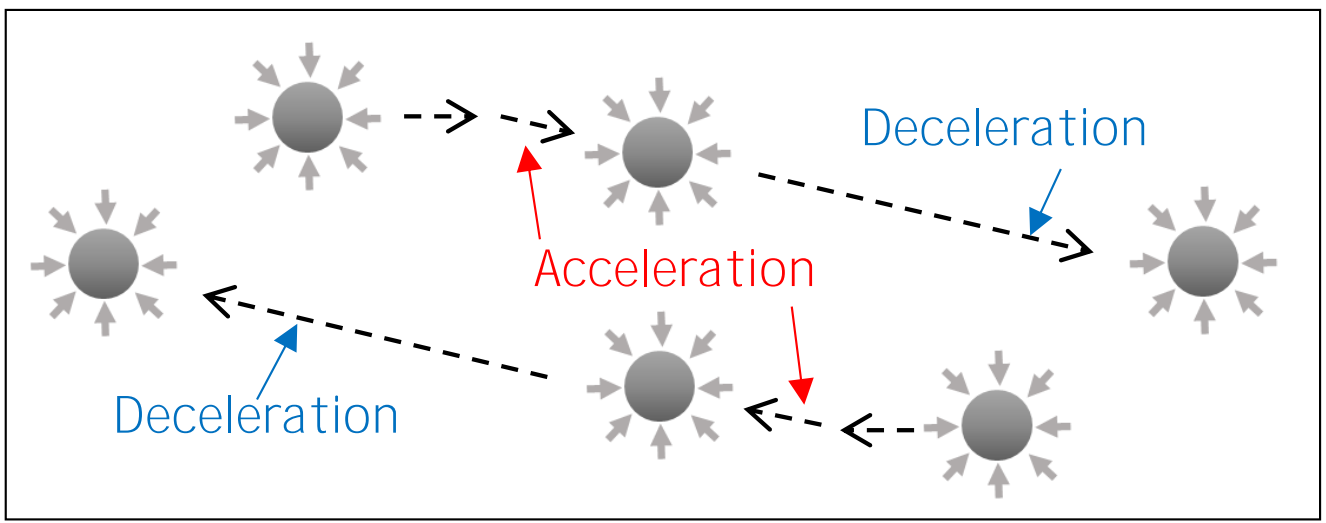

Fig. S2. Gravitational forcefields interaction between passingby planets 


\section{References:}

Wells, A. F. Structural Inorganic Chemistry (Oxford, 2012). ISBN-10: 0198553706.

2 Basdevant, J.-L.; Rich, J. and Spiro, M. Fundamentals in nuclear physics (Springer, 2005). ISBN 0-387-01672-4.

3 Fewell, M. P. The atomic nuclide with the highest mean binding energy, Am. J. Phys. 63 (7), 653-658 (1995). doi.org/10.1119/1.17828.

4 Tian, Z. Nanoparticles' and Atoms' Geometry-Wave Potential Unified Properties. ChemRxiv. Preprint (Sept. 5, 2019). https://doi.org/10.26434/chemrxiv.9759551.v1.

5 Tian, Z. Geometry-Wave Potential Quantified and Unified Properties in General Chemistry. ChemRxiv. Preprint (Sept. 5, 2019). https://doi.org/10.26434/chemrxiv.9759581.v1. 